\section{Moderne Therapiekonzepte für das idiopathische Parkinson-Syndrom}

Heinz Reichmann

Klinik und Poliklinik für Neurologie, Universitätsklinikum Carl Gustav Carus, Technische Universität Dresden

psychoneuro 2003; 29 (10): 442-448

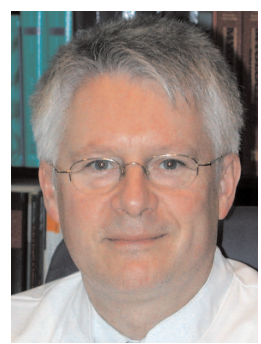

Heinz Reichmann
Die Therapie des idiopathischen Parkinson-Syndroms wird zunächst in die Behandlung der Frühphase und der Spätphase sowie in die Behandlung biologisch junger oder alter Patienten unterteilt. Biologisch junge de-novo-Patienten sollten mit Dopamin-Agonisten oder mit Amantadin und Selegilin oder mit allen drei Präparaten gleichzeitig behandelt werden. Bei de-novoPatienten, die biologisch alt sind, wird man Levo-Dopa mit einem COMT-Hemmer und falls möglich auch einen Dopamin-Agonisten verwenden. In fortgeschrittenen Phasen der Erkrankung wird man ebenfalls die Kombination aus Levo-Dopa/COMT-Hemmer und einem Dopamin-Agonisten wählen. Bei Patienten mit medikamentös nicht beherrschbaren Dyskinesien bietet sich die tiefe Hirnstimulation (oder zukünftig die Implantation dopaminerger Retinazellen?) an. Aufgrund der unzureichenden Datenlage, kann die Transplantation dopaminerger embryonaler Zellen heute noch nicht empfohlen werden. Auf den Einsatz von Stammzellen wird man noch warten müssen.

$\mathrm{M}$ eilensteine in der Entwicklung einer effektiven Therapie des Parkinson-Syndroms waren Belladonna, LevoDopa mit Decarboxylase-Hemmung, die Einführung des ersten DopaminAgonisten Pravidel, die MAO-Hemmer, die COMT-Hemmer, die Antiglutamatergika und in letzter Zeit wieder vermehrt die operativen Verfahren.

Die Entwicklung der modernen Parkinson-Therapie besteht aus zufälligen Entdeckungen über die Wirksamkeit von Substanzen wie z.B. Amantadin oder aus gezielten Entwicklungen wie z.B. die Einführung von Nichtergolin-Dopaminagonisten.

Während man früher noch glaubte, dass die Parkinson-Erkran- kung eine reine Dopamin-MangelKrankheit ist, weiß man heute, dass auch das glutamaterge, adrenerge, noradrenerge, serotonerge, cholinerge und wohl weitere Transmittersysteme in ihrer Balance gestört sind. Aus der Grundlagenforschung sind darüber hinaus neue Erkenntnisse zur Entstehung des nigralen Zelltodes bekannt (Apoptose, oxidativer Stress, Komplex-I-Mangel der Atmungskette, Synukleinopathie, Proteinabbaustörung, entzündliche Vorgänge, u.v.a.), so dass es aus meiner Sicht vermessen wäre, darauf zu hoffen, dass ein einzelnes Medikament alle Mechanismen korrigiert und somit zur Behandlung multipler Transmitterstörungen ausreichen würde. Dies bedeutet, dass es zum Konflikt mit einer preisdiktierten Verschrei- bungspolitik kommen muss. Die Therapievorschläge in diesem Artikel erscheinen zwar zunächst kostenintensiv, sind aber aus meiner praktischen Erfahrung heraus ein Garant dafür, dass die Patienten überwiegend und lange ambulant behandelt werden können und auch bei entsprechendem Alter arbeitsfähig bleiben können. Aus Arbeiten von Dodel und Mitarbeitern (2) ist klar ersichtlich, dass die Patienten in den Spätstadien ungleich "teurer" werden als in den Frühstadien, so dass es nicht nur aus ärztlich-ethischen Gründen, sondern bei genauerem Nachdenken auch aus ökonomischen Gründen ratsam ist, die Frühphasen der Krankheit optimal, frei von jeglichem Kostendenken, zu behandeln.

\section{Frühbehandlung}

Wichtige Ziele der Frühbehandlung sind die Unterdrückung der Symptome (symptomatische Kontrolle), das Vermeiden von LevoDopa-Spätfolgen durch sparsamen Umgang mit Levo-Dopa und eventuell die bevorzugte Verwendung von Medikamenten mit möglicher Neuroprotektion.

Während in den letzten Jahren noch die einhellige Meinung bestand, dass der Einsatz von LevoDopa den besten symptomatischen 
Erfolg bei den geringsten Nebenwirkungen garantiert, kann dies heute als widerlegt gelten. So haben z.B. Rascol und Kollegen (8) in einem Doppel-blind-Design nachweisen können, dass in den Hoehn und Yahr Stadien I und II der Dopamin-Agonist Ropinirol genauso effektiv ist wie Levo-Dopa. Aus dieser und ähnlichen Studien folgert man, dass Dopamin-Agonisten zur Frühbehandlung der Parkinson-Krankheit eingesetzt werden sollten. Ein weiterer Grund dafür ist die Tatsache, dass Dopamin-Agonisten bei weitem weniger Dyskinesien auslösen als LevoDopa. Ähnlich wie in der Epileptologie und der Depressionsbehandlung muss für jeden einzelnen Patienten aufgrund seines Verträglichkeitsprofils, seiner biografischen Daten und seiner Ko-Morbidität individuell der best geeignete Dopamin-Agonist ausgewählt werden. Es stehen uns dafür kurz wirksame (Apomorphin, Lisurid), extrem langwirksame (Cabergolin) und Agonisten mit einer mittellangen Halbwertszeit zur Verfügung. Außerdem sind DopaminAgonisten mit unterschiedlichen Rezeptoraffinitäten im Handel (Tab. 1). Ein weiterer wichtiger Gesichtspunkt für die Auswahl von Dopamin-Agonisten ist ihre unterschiedlich lange Eindosierzeit (Tab. 2). Man muss den Eindruck gewinnen, dass die Marktführer in Deutschland die Dopamin-Agonisten sind, die sich nach einem besonders einfachen Schema aufdosieren lassen. In absehbarer Zeit werden Dopamin-Agonisten nicht nur oral, sondern auch als Pflaster und als slow-release Präparate zur Verfügung stehen.

Noch nicht entschieden ist die Frage, ob Dopamin-Agonisten wie
Ropinirol und Pramipexol wegen ihres möglichen neuroprotektiven Ansatzes bevorzugt verwendet werden sollten. Für beide Substanzen konnte nuklearmedizinisch eine um ca. 7\% niedrigere jährliche Verlustrate an dopaminergen Neuronen im Vergleich zu Levo-Dopa gezeigt werden. Es ist dabei wegen eines jeweils fehlenden Plazebo-Armes aber nicht geklärt worden, ob der DopaminAgonist neuroprotektiv oder LevoDopa neurotoxisch ist oder ob beide Möglichkeiten zutreffen. Weiter gibt es Kritiker, die bemängeln, dass die Dopamin-Agonisten evtl. mit den Rezeptoren so interagieren, dass Kunstprodukte gemessen wurden. Es wird daher notwendig, dieser Frage nachzugehen, obwohl Spezialisten wie Brooks (1) davon ausgehen, dass sie die tatsächlich vorhandene Situation mit ihren nuklearmedizinischen Methoden widerspiegeln.

Während der Einsatz von Dopamin-Agonisten zur Vermeidung von Dyskinesien in der Frühphase der Behandlung unumstritten sind (6, 9), ist die Situation bzgl. der MAO-BHemmer wesentlich kontroverser. Die DATATOP-Studie hat gezeigt, dass durch den frühen Einsatz von Selegilin der Beginn von Levo-Dopa um neun Monate verschoben werden kann. Man geht heute aber davon aus, dass es sich dabei um eine symptomatische und nicht neuroprotektive Wirkung von Selegilin handelt. Nachdem die Substanz im Labor aber eindeutig neuroprotektive Eigenschaften aufweist, wurden zwei Studien aufgelegt, die nachwiesen, dass Patienten, die neben LevoDopa oder einem Dopamin-Agonisten noch Selegilin erhielten, nach
Absetzen aller Präparate länger beweglich blieben. Außerdem gibt es neue Überlegungen bzgl. der Halbwertzeit von Selegilin (4). Ich verwende daher weiterhin Selegilin in der Frühphase der Parkinson-Krankheit, um Dopaminergika zu sparen und eine mögliche Neuroprotektion für den Patienten nicht zu verpassen.

An den "medium spiny neurones“ des Striatum herrscht eine Dysbalance zwischen einer dopaminergen Unterstimulation und einer glutamatergen Überstimulation, so dass das Konzept des frühen Einsatzes von Amantadin oder Budipin (Cave: Sondervorschriften wegen Gefahr der Entstehung von torsades de pointes) ein gewichtiges ist. Amantadin ist sehr gut wirksam gegen Rigor und Bradykinese und verhindert Dyskinesien, Budipin ist besonders gut gegen Tremor wirksam, so dass der Einsatz eines der beiden Antiglutamatergika von Seiten der Symptomverbesserung hilfreich ist. Dazu kommt zumindest für das Amantadin die theoretische Möglichkeit der Neuroprotektion, zumindest indirekt dadurch, dass der NMDA-Rezeptor gehemmt wird und somit kein Kalzium in die Zelle fließen kann und deren Untergang mit auslösen kann. Gelegentlich wird initial eine Dopamin-Agonisten-Monotherapie oder eine Kombination aus Amantadin und Selegilin propagiert. Nachdem es keine Vergleichsstudien dazu gibt, muss offen bleiben, was die optimale Initialtherapie ist. Nach wissenschaftlichen Gesichtspunkten spricht nichts gegen eine initiale Kombination mit allen drei Substanzen, um sie niedrig einsetzen zu können und so ein

\section{Tab. 1 Dopamin-Agonisten und ihre pharmakokinetischen Eigenschaften}

\begin{tabular}{lllll} 
Dopamin-Agonist & $\mathbf{D}_{\mathbf{1}}$ Rezeptor & $\mathbf{D}_{2}$ Rezeptor & $\mathbf{D}_{\mathbf{3}}$ Rezeptor & Plasma-Halbwertszeit \\
\hline Dopamin & + & +++ & + & 1,5 Stunden \\
\hline Apomorphin & + & ++ & ++ & 20 Minuten \\
Pergolid & + & +++ & ++ & 18 Stunden \\
\hline Bromocriptin & - & +++ & + & 3 Stunden \\
\hline Lisurid & Partiell + & +++ & $?$ & Stunden \\
\hline$\alpha$-DH-Ergocriptin & Partiell + & ++ & +++ & 6 Stunden \\
Ropinirol & - & +++ & +++ & 8 Stunden \\
\hline Pramipexol & - & + & 68 Stunden \\
\hline Cabergolin & Partiell/+ & +++ & & ++
\end{tabular}


günstiges Nebenwirkungsprofil bei additiv guten symptomatischen Effekten zu erzielen (Abb. 1).

\section{Behandlung in späteren \\ Stadien}

In späteren Stadien der Erkrankung oder bei Patienten mit hoher Komorbidität wird Levo-Dopa zum Einsatz kommen. Häufig wird es gelingen, Levo-Dopa mit einem Agonisten zu kombinieren, so dass Levo-Dopa möglichst lange nicht höher als mit 400-600 mg/Tag dosiert werden muss und somit das Dyskinesie-Risiko überschaubar ist. Levo-Dopa hat eine Halbwertszeit von ca. 1,5 bis 2,5 Stunden, so dass es eine phasische Stimulation des Dopamin-Rezeptors bedingt, wohingegen die modernen Dopamin-Agonisten mit ihrer deutlich längeren Halbwertzeit eine tonische Stimulation bewirken. Chase geht davon aus, dass die tonische Stimulation des Dopamin-Rezeptors die Voraussetzung für die Verhinderung von Dyskinesien ist - ein Konzept, das allerdings nicht allein richtig sein kann, da auch der Einsatz von Apomorphin, das eine Halbwertszeit von lediglich 20 Minuten hat, zu wenig Dyskinesien führt. Die Wirkung von Levo-Dopa kann durch COMTHemmer verlängert werden, womit wearing-off verkürzt und verhindert werden können. Sobald die Daten der Triple-Tablette vorliegen werden (Tablette mit Levo-Dopa, Decarboxylase-Hemmer und Entacapon) wird es spannend werden, zu sehen, ob dies zu einem weiteren Vorteil bzgl. Wirkung und Dyskinesie-Vermeidung führt.

\section{Tiefe Hirnstimulation}

Die nächsten Jahre werden uns auch hoffentlich Daten zur Frage liefern, ob die tiefe Hirnstimulation, wie es bis jetzt der Fall ist, erst bei medikamentös nicht mehr korrigierbaren Hyperkinesien und Parkinsonsymptomen oder bereits früher eingesetzt werden sollte. Unsere eigenen Erfahrungen untermauern, dass es sich um eine hoch potente Möglichkeit handelt, schwere Dyskinesien, die man auch durch Amantadin und DopaminAgonisten-Hochdosistherapie (5) nicht mehr korrigieren kann, meist fast komplett zum Sistieren zu bringen. Es muss betont werden, dass nicht nur die Operation "gekonnt“ sein muss, sondern dass auch im Vorfeld und in der Nachbetreuung z.B. beim Einstellen der Stimulationsparameter oder beim Festsetzen der Begleitmedikation ein sehr erfahrener Parkinson-Stimulations- spezialist vor Ort sein muss. Einer Ausweitung der Methode auf immer kleinere Zentren sind daher Grenzen gesetzt.

\section{Transplantation von dopaminergen embryonalen Zellen}

Die Transplantation von dopaminergen embryonalen Zellen hat sich in Deutschland im Gegensatz z.B. zu Schweden nicht etabliert. Zu groß sind die Bedenken gegen die Tatsache, dass zur Transplantation von genügend mesenzephalen Zellen acht Schwangerschaften gleichzeitig unterbrochen werden müssen. Zwei Publikationen aus der jüngsten Zeit $(3,7)$ scheinen zu beweisen, dass die Methode nur Patienten unter dem 60. Lebensjahr hilft und dies auch nur in manchen Fällen. Ein gänzlich anderer Ansatz wird darin bestehen, aus der Retina eines verstorbenen Frühgeborenen gewonnene dopaminerge Zellen mittels Mikrogelatine-Partikel zu vermehren und dann zu implantierten. Erste Erfolge konnten bei Patienten verzeichnet werden, die schon vor zwei Jahren operiert wurden (10).

\section{Stammzell-Therapie}

Besondere Zurückhaltung scheint bei der Abschätzung der Er-

Tab. 2 Dosierung in $\mathrm{mg} /$ Titration

\begin{tabular}{|c|c|c|c|c|c|c|c|}
\hline & Bromocriptin & Cabergolin & $\alpha$-DHEC & Lisurid & Pergolid & Pramipexol & Ropinirol \\
\hline 1. Woche & $1 \times 1,25$ & $1 \times 1,0$ & $2 \times 5$ & $1 \times 0,1$ & Startpackung & $3 \times 0,088$ & $3 \times 0,25$ \\
\hline 2. Woche & $2 \times 1,25$ & $1 \times 1,0$ & $2 \times 5$ & $2 \times 0,1$ & $1 \times 0,125+2 \times 0,25$ & $3 \times 0,18$ & $3 \times 0,5$ \\
\hline 3. Woche & $3 \times 1,25$ & $1 \times 2,0$ & $2 \times 10$ & $3 \times 0,1$ & $1 \times 0,25+2 \times 0,5$ & $3 \times 0,36$ & $3 \times 0,75$ \\
\hline 4. Woche & $2 \times 1,25+1 \times 2,5$ & $1 \times 2,0$ & $2 \times 10$ & $2 \times 0,1+1 \times 0,2$ & $1 \times 0,5+2 \times 1,0$ & & $3 \times 1,0$ \\
\hline 5. Woche & $1 \times 1,25+2 \times 2,5$ & $1 \times 3,0$ & $2 \times 15$ & $2 \times 0,2+1 \times 0,1$ & & & $3 \times 2,0$ \\
\hline 6. Woche & & & $2 \times 15$ & $3 \times 0,2$ & & & $3 \times 3,0$ \\
\hline 7. Woche & & & $2 \times 20$ & $2 \times 0,2+1+0,3$ & & & \\
\hline 8. Woche & & & $2 \times 20$ & $3 \times 0,25$ & & & \\
\hline 9. Woche & & & $1 \times 10+2 \times 20$ & $2 \times 0,25+1 \times 0,5$ & & & \\
\hline 10. Woche & & & $1 \times 10+2 \times 20$ & $2 \times 0,5+1 \times 0,25$ & & & \\
\hline 11. Woche & & & $3 \times 20$ & & & & \\
\hline $\begin{array}{l}\text { Durchschnittliche } \\
\text { Tagesdosis (mg) } \\
\text { erreicht ab Woche }\end{array}$ & 8 & 5 & 11 & 10 & 5 & 3 & 6 \\
\hline $\begin{array}{l}\text { Durchschnittliche } \\
\text { Tagesdosis (mg) }\end{array}$ & 15 & 3,0 & 60 & 1,25 & 2,5 & 1,08 & 9,0 \\
\hline $\begin{array}{l}\text { Maximale } \\
\text { Tagesdosis (mg) }\end{array}$ & 30 & 6,0 & 120 & 2,0 & 5,0 & 3,3 & 24 \\
\hline $\begin{array}{l}\text { Dosisbereich } \\
\text { (mg) pro Tag }\end{array}$ & $12,5-30$ & $2,0-6,0$ & $40-120$ & $0,6-2,0$ & $0,75-5,0$ & $0,246-3,3$ & $3-24$ \\
\hline
\end{tabular}




\section{Abb. 1 Entscheidungsbaum zur Therapie des idiopathischen Parkinsonsyndroms}

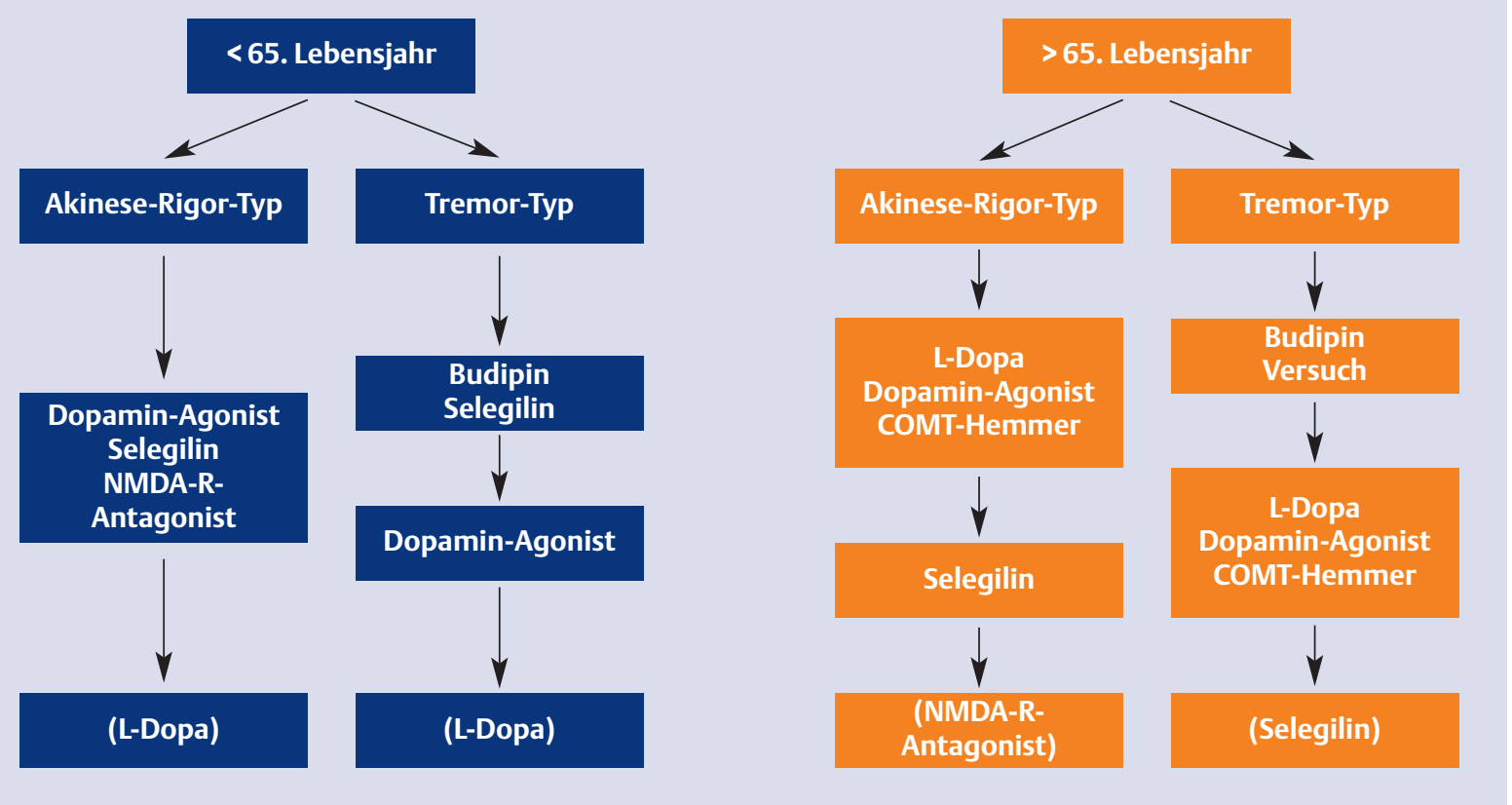

folgsaussichten der Stammzell-Therapie angezeigt. Unstrittig ist die Parkinson-Krankheit mit ihrem Dopaminmangel eine Modellkrankheit, um durch die Implantation von Dopamin-produzierenden Stammzellen eine Korrektur und vielleicht Heilung dieser Krankheit zu erzielen. Problematisch ist beim Einsatz von embryonalen Zellen aber die Gefahr der Abstoßung oder der Entwicklung von Teratomen, wie dies in Tierversuchen der Fall war. Beim Einsatz von embryonalen oder adulten Stammzellen muss weiter abgewartet werden, wie sich die Implantation von ungesteuert Dopaminproduzierenden Zellen auf die Symptome des Patienten auswirken wird. Es ist anzunehmen, dass man mit der Implantation lediglich die Basisversorgung gewährleisten darf, um keine Hyperkinesien auszulösen. Somit werden auch diese Patienten individuell auf ihre Bedürfnisse angepasste Medikationen erhalten müssen. Dabei ist noch unklar, ob/wie Stammzellen und Medikamente interagieren werden. Stammzellen des Patienten zu gewinnen und zu Dopamin-produzierenden Neurone $z u$ transformieren, erscheint auf den ersten Blick vielversprechend, könnte aber das Problem mit sich bringen, dass sogar Stamm- zellen die Ursache der ParkinsonKrankheit in sich bergen. Derzeit kann den Patienten somit m. E. noch keine große Hoffnungen auf die Stammzelltherapie gemacht werden.

\section{Ausblick}

Was wird die Zukunft noch bringen? Wir werden sicherlich durch die Beeinflussung neuer Transmittersysteme versuchen, der Komplexität der Parkinson-Krankheit therapeutisch noch besser gerecht $\mathrm{zu}$ werden. Vielleicht werden der Einsatz von Coenzym $Q$ oder von Immunophilinen kleine Fortschritte bringen, man wird weitere MAO-BHemmer mit zumindest im Labor noch besserer Neuroprotektion zulassen und eine neue noch besser lösliche Levo-Dopa-Formulierung auf den Markt bringen. Aber eine Wunderdroge ist meines Wissens immer noch nicht in der "Pipeline“. Wir werden uns daher weiter bemühen müssen, mit den vielen uns glücklicherweise zur Verfügung stehenden Medikamenten unsere Patienten nach bestem Erkenntnisstand zu therapieren. Die Therapie des idiopathischen Parkinsonsyndroms ist eine Herausforderung, aber gleichzeitig auch eine große Freude, wenn man daran denkt, wie weit die Möglichkeiten der positiven Einflussnahme auf die Symptome im Vergleich zu allen anderen neurodegenerativen Erkrankungen schon gediehen sind. Gerade diesen hoffnungsvollen Aspekt muss man den Patienten immer wieder vermitteln und durch die optimierte Therapie unterstreichen.

Modern Therapeutic Concepts for Idiopathic Parkinson's Disease Management of idiopathic Parkinson's disease is differentiated first into treatment of the early phase and of the late phase, and then again into care of biologically younger and biologically older patients. Biologically young de-novo patients should be treated with dopamine agonists or amantadine and selegiline, or with all three substances simultaneously. In the case of biologically old de-novo patients levodopa in combination with a COMT inhibitor and, if possible, also a dopamine agonist, will be used. In advanced stages of the disease, again the combination of levodopa/ COMT inhibitor and a dopamine agonist will be employed. In patients with dyskinesias intractable to drug treatment, deep brain stimulation (or, in future, implantation of dopaminergic retinal cells?) remains an option. In light of the inadequacy of 
available data, the transplantation of dopaminergic embryonic cells cannot at present be recommended, and the use of stem cells is something for the future.

Key Words: idiopathic Parkinson's disease - levodopa - amantadine dopamine agonists - COMT inhibitors - MAO B inhibitors

\section{Literatur}

1. Brooks DJ. Imaging end points for monitoring neuroprotection in Parkinson's disease. Ann Neurol 2003; 53 (Suppl 3): S110-S119

2. Dodel RC, Berge K, Oertel WH. Health-related quality of life and healthcare utilisation in patients with Parkinson's disease: impact of motor fluctuations and dyskinesias. Pharmacoeconomics 2001; 19: 1013-1038 3. Freed CR, Greene PE, Breeze RE, Tsai WY, Du-Mouchel W, Kao R, Dillon S, Winfield H, Culver S, Trojanowski JQ, Eidelberg D, Fahn S. Transplantation of embryonic dopamine neurons for severe Parkinson's disease. N Engl J Med 2001; 344: 710-719

4. Gerlach M, Reichmann H, Riederer P. Die Parkinson Krankheit.Wien New York, Springer Verlag, 2003

5. Müngersdorf M, Sommer U, Sommer M, Reichmann H. High-dose therapy with ropinirole in patients with Parkinson's disease. J Neural Transm 2001; 108: 1309-1317 6. Olanow CW, Watts RL, Koller WC. An algorithm (decision tree) for the management of Parkinson's disease (2001): teatment guidelines. Neurology 2001; 56 (Suppl 5): S1-S88 7. Olanow C. Transplantation for Parkinson's disease: Pros, cons, and where do we go from here? Movement Disord 2002; 17(Suppl 5) S15

8. Rascol O, Brooks D, Korczyn AD, De Deyn PP, Clarke CE, Lang AE, for the 056 Study Group. A five-year study of the incidence of dyskinesia in patients with early Parkinson's disease who were treated with ropinirole or levodopa. N Engl J Med 2000; 342: 1484-1491

9. Reichmann H, Sommer U, Fuchs G, Hefter H, Mark G, Müller T, Thümler R, Ulm G, Vieregge $P$. Drug treatment guidelines for the long-term management of Parkinson's disease. J Neurol 2000; 247 (Suppl 4): 40-41 10. Watts RL, Raiser CD, Stover NP, Cornfeldt ML, Schweikert AW, Subramanian T, Bakay RAE. Stereotaxic intrastriatal implantation of retinal pigment epithelial cells attached to microcarriers in advanced Parkinson disease. Neurology 2001; 56(Suppl 3): A283

\section{Korrespondenzadresse:}

Prof. Dr. Heinz Reichmann

Klinik und Poliklinik für Neurologie, Universitätsklinikum Carl Gustav Carus, Technische Universität Dresden

Fetscherstraße 74

01307 Dresden
01.-02.11.03

10. Jahrestagung des AKF „Lust auf Dialog - Fruengesundheit und Migration“

Ort: Berlin, Rathaus Schöneberg Veranstalter: Arbeitskreis Frauengesundheit in Medizin, Psychotherapie und Gesellschaft e.V. Information/Anmeldung: AKF e.V., Knochenhauerstr. 20/25, 28195 Bremen, Tel. 04 21/4 3493 40, Fax: 04 21/1 6049 60, www.akf-info.de

\section{3. -05.11 .03$}

Fachkonferenz „Methodenwissen in der Suchtkrankenhilfe“

Ort: Koblenz

Information/Anmeldung: Deutsche

Hauptstelle gegen die Suchtgefahren e.V., Postfach 1369, 59003 Hamm, Tel.: 023 81/90 15-0, Fax: 023 81/ 9015 31, E-Mail: kaldewei@dhs.de, Internet: www.dhs.de

06.11 .03

Jahrestagung der Deutsch-Niederländischen-Gesellschaft für Psychiatrie „Soziale Psychiatrie in (a)sozialen Zeiten“"

Ort: Westfälische Klinik für Psychiatrie und Psychotherapie Münster Information/Anmeldung: Sekretariat Prof. Trenckmann,

Tel.: 023 72/8 61-1 09 und MediScience, Medizinischer Dokumenten- und Kongressservice, Tel.: 02 31/52 13 72, E-Mail: medi@mediscience.de, www.medi-science.de

19.-23.11.03

Kongress der Deutschen Gesellschaft für Psychiatrie, Psychotherapie und Nervenheilkunde (DGPPN)

Ort: Berlin

Informationen/Anmeldung: CPO HANSER SERVICE, Paulsborner Str. 44, 14195 Berlin, Tel: 0 30/ 30066 90, Fax: 0 30/ 30573 91, E-Mail: berlin@cpo-hanser.de, www.dgppn-kongress2003.de

13.12.03

9. Weissenauer Epilepsie-Tagung Ort: Weissenau Information/Anmeldung: Sekretariat der Abteilung für Neurologie und Epileptologie. Die Weissenau, Weingartshoferstraße 2, 88214 Ravensburg, Tel.: 07 51/76 01-23 90
09.-13.02.04

\section{International Congress of} Biological Psychiatry

Ort: Sydney Convention \& Exhibition

Centre, Sydney, Australia

Sekretariat: wfsbp@icms.com.au Homepage:http://www.icms.com. au/wfsbp2004, ICMS Pty Ltd, 84 Queensbridge Street, Southbank, Victoria 3006, Australia, Tel.: +61 3 96820244 , Fax: +61 396820288

\subsubsection{4}

Symposium „Karl Leonhard 100 Jahre. Psychiatrie und Neurologie im Wandel?"

Ort: Berlin

Information: Univ.-Prof. Dr. K.-J. Neumärker, Chefarzt Klinik für Kinder- und Jugendpsychiatrie/ Psychiatrie, DRK-Kliniken Westend/ Berlin, Spandauer Damm 130, 14050 Berlin,

E-Mail: k.-j.neumaerker@drkkliniken-westland.de, Tel.: 0 30/ 30 35-45 15, Fax: 0 30/30 35-45 19

14.- 18.04 .04

$12^{\text {th }}$ AEP (Association of European Psychiatrists) Congress

Ort: Genf, Schweiz

Deadline für Abstracts: 15. November 2003

Kongressorganisation/Wissenschaftliches Sekretariat: http://www. kenes.com/ 17 Rue du Cendrier, PO Box 1726,CH-1211 Geneva 1, Switzerland, Tel: +41 229080488 , Fax: +41 22732 2850,

E-mail: aep12@kenes.com

\section{1.-06.05.04}

\section{APA (American Psychiatric} association) Annual Meeting Ort: Javits Convention Center New York, NY Information/Anmeldung: www.psych.org

\section{3.-0.4.06.04}

Tagung „Der Körper in der tiefenpsychologisch fundierten und analytischen Psychotherapie“ Ort: Rotenburg Informationen/Anmeldung: Sekretariat der Klinik für Psychiatrie und Psychotherapie Rotenburg, Frau Klause, Tel.: 042 61/77 67 00, Fax: 042 61/77 67 03, Postfach 1211, 27342 Rotenburg 